\title{
Optical properties of SPPs on low-dimensional metal structures and their sensing applications
}

\author{
Zhaohua Wang ${ }^{1,}$, Liqing Ren ${ }^{1}$ \\ ${ }^{1}$ College of Energy Engineering, Yulin University, Shanxi 719000, China \\ awangzhaohua841102@163.com
}

Keywords: low-dimensional metal; plasmon polariton; metal nanowire

\begin{abstract}
Surface plasmon polaritons (SPPs) on low-dimensional metal materials are unique in properties. This enables SPPs to play a role in some special fields, notably high-precision sensing. On the basis, this paper attempts to analyze the optical properties of SPPs on low-dimensional metal structures and their sensing applications. Then, metal nanowire cases are adopted to further illustrate such applications. To sum up, this paper attempts to clarify advantages of low-dimensional metals, thus providing theoretical support for the follow-up applications.
\end{abstract}

\section{Introduction}

Research findings, either in the past or at present, have indicated a brilliant application prospect of low-dimensional metals. More and more attention has been paid to tapping the commercial value of low-dimensional metals. On the whole, low-dimensional metal materials show favorable performance in controlling and restricting sub-wavelength light. This lays a solid research foundation for a pending issue long existing in the research field of optics and chemistry. The current achievements can also be further optimized under the support of new technologies. The super-compact full-optical circuits, laser technology, nonlinear research, biological research and quantum optics-all this scientific research work can benefit from development of the low-dimensional metals.

\section{Optical properties of SPPs on low-dimensional metal structures}

Low-dimensional metals refer to various metal materials whose dimensionality is smaller than three. Zero-dimensional metal materials, one-dimensional metal materials and two-dimensional metal materials-all these fall into the category of low-dimensional metals. Table 1 shows by what standards different low-dimensional metals are divided.

Table 1. Standards to divide low-dimensional metal materials

\begin{tabular}{cl}
\hline Dimensionality & Standards \\
\hline Zero-dimensional & Quantum dots, composed of a few molecules or atoms; nanoscale \\
One-dimensional & $\begin{array}{c}\text { Quantum wires; the wire thickness is nanoscale } \\
\text { Two-dimensional }\end{array}$ \\
& $\begin{array}{c}\text { Several faces of the thin film or two materials; the boundary depth and the } \\
\text { thin film thickness are nanoscale. }\end{array}$ \\
\hline
\end{tabular}

To modern physics, chemistry and biology, applications of low-dimensional metals are of vital significance. This cannot be separated from optical properties of SPPs on low-dimensional metal structures. SPPs on metal structures refer to elementary excitations caused by constant collisions, responses and vibrations of charged particles on the free-state metal surface. The photons then interact with the SPPs to generate new properties of elementary excitations via coupling. The new properties here are actual the optical properties of SPPs on low-dimensional metal structures. From the above description, it can be easily observed that electrons lay the foundation of reactions, and the electronic components are shown in Table 2. 
Table 2. Basic electronic components

\begin{tabular}{ll}
\hline Materials & Basic situations \\
\hline Orbitans & Quasi-particles; appear when electronic charges spin to get separated. \\
Holons & Quasi-particles; appear when electronic charges spin to get separated. \\
Spinons & Also known as electric charges; one kind of quasi-particles; appear when \\
& electronic charges spin to get separated. \\
\hline
\end{tabular}

To be specific, there are many charged particles on the metal surface. When the external optical field appears, the distribution of the charged ions will be changed, upsetting the mutual balance. Then, oscillation occurs. To low-dimensional metal materials, the charge oscillation is conducive to the sub-wavelength energy control, and can significantly strengthen its spatial and near field properties. This helps get rid of the traditional optical diffraction limitations, making nanoscale optical control a possibility. Take enhanced properties of SPPs on low-dimensional metals for example. These properties can significantly contribute to mutual interaction between the enhanced light and certain material. Based on this, the nonlinear optical phenomena can be further studied. The sub-wavelength energy control can achieve a better understanding of the low-dimensional metal surface information to improve the optical instrument manufacturing [1].

Conductive metals are a kind of low-dimensional metals, and one of the most commonly-seen application types. SPPs on conductive metals are a kind of compound electromagnetic waves, which exist on the boundary between two materials, such as the conductive metal and the air dielectric, whose dielectric constant symbol is opposite to each other. In general, SPPs on conductive metals are between the metals and the air dielectric. As long as the frequency remains unchanged, the relationship between the optical momentum in the free space and the momentum of SPPs on conductive metals follows distinct rules. In other words, mismatch exists between the two. If the surface of conductive metals is highly smooth and flat, the light source is required to compensate the momentum during the boundary plasmon polariton excitation. The specific compensation standards are decided by degree of smoothness and flatness of the conductive metal surface. The basic relationship between the two is shown in Table 3.

Table 3. Relationship between the optical momentum and the momentum of SPPs on conductive metals

\begin{tabular}{|c|c|c|}
\hline Momentum type & Relationship & Compensation state \\
\hline Momentum of the light & $\begin{array}{l}\text { Comes in } \\
\text { through } \\
\text { incidence }\end{array}$ & To compensate \\
\hline $\begin{array}{l}\text { Momentum of SPPs on conductive } \\
\text { metals }\end{array}$ & Accepts & To be compensated \\
\hline
\end{tabular}

\section{Sensing applications of low-dimensional metal}

\subsection{Biochemical sensing}

Metal nanoparticles have a great role to play in a changing dielectric environment. That the resonance frequency is highly sensitive to changes creates room for realization of the small-size biochemical sensing. In principle, test of the resonance peak movement caused by the environmental refractive index can help gain a better understanding of the resonance peak changes at any time or any period of time, based on which sensing can be realized. Compared with traditional technologies, the metal nanoparticle sensing technology can significantly improve accuracy as shown in Table 4. 
Table 4. Comparison between the metal nanoparticle sensing technology and traditional technologies

\begin{tabular}{lll}
\hline Technological type & Response time (s) & Numerical accuracy \\
\hline Metal nanoparticle sensing technology & Below 0.06 & $\begin{array}{c}\text { Correct to five decimal } \\
\text { places } \\
\text { Correct to three } \\
\text { Traditional technologies }\end{array}$ \\
decimal places
\end{tabular}

In 2013, Western scholars conducted an experiment. The metal nanoparticle hydrogen sensing featuring a SiOx core-shell structure was adopted. The low-dimensional metal particle was placed on the substrate, and the substrate was plated with a 10 to 15 nanometer thick target. Later, researched changed the concentration of seaside hydrogen. The palladium and the hydrogen reacted with each other to form PdH. The refractive index was thus changed, and the metal nanoparticles showed an obvious blue shift in the harmonic peak. The detection limit reached the level of $0.3 \%$, and the repeatability was favorable [2]. Based on this, researchers synthesized a palladium nanoparticle with a diameter of around 4 nanometers, and covered it on the golden nanorod surface. After that, the hydrogen sensing was further monitored. Through the hydrogen concentration adjustment, the nanorod dark field refractive spectrum was captured. More details are shown in Table 5.

Table 5. Changes of the nanorod dark-field refractive spectrum at different hydrogen concentrations

\begin{tabular}{ccl}
\hline Materials & $\begin{array}{c}\text { Hydrogen } \\
\text { concentration (\%) }\end{array}$ & Changes of color wavelengths \\
\hline \multirow{3}{*}{ Nanorod } & 10 & Short \\
& 20 & Lengthened \\
& 30 & Further lengthened \\
\hline
\end{tabular}

3.2 Nanowires and nanofiber sensing

Surface of low-dimensional metals represented by conductive metals has been studied for more than 110 years. As to power transmission in the earliest electric system, scholars once put forward the idea of cylindrical wire transmission. Nevertheless, limited by the technological level, the conception was confined to the theoretical level. Later, scholars had made attempts to apply it to other fields. Currently, the major technological restriction is to find a suitable excitation source. In 2012, relevant researchers chose nanofibers and nanowires for coupling to generate the excitation source. The wavelength was set to be 650 nanometers and the coupling length was set to be 220 nanometers. After detection, it was found that if the nanowires were silver, the coupling rate could be well above $80 \%$. In 2013, researchers further optimized the nanometer wavelength and the coupling length. Under the prerequisite of not changing the nanowire materials, the wavelength was adjusted to 785 nanometers and the nanowires to 280 nanometers. After repeated experiments, it was found out that the photon and plasmon polariton exchange efficiency was above $91 \%$ on average. This meant efficiency of optical fiber sensing could be significantly improved. However, due to technological difficulties facing manufacturing and mass applications of silver nanowires, the sensing system has not yet got fully promoted.

\section{Conclusions}

This paper focuses on analyzing optical properties and sensing applications of SPPs on low-dimensional metal structures. Research findings suggest quick response and regular optical compensation constitute two major optical properties of SPPs on low-dimensional metals. Relying on these properties, low-dimensional metals can play a greater role in the sensing field, including biochemical sensing, nanowire sensing and nanofiber sensing. In particular, its quick response can facilitate some relevant work. In the future, more attention should be paid to a full utilization of 
optical properties of SPPs on low-dimensional metals so as to contribute to optimization of various sensing systems.

\section{Acknowledgements}

This work was supported by High level talent introduction and research start-up fund(11GK6I); Shaanxi science and technology research and development plan project(2014K05-11)

\section{References}

[1]Zhang Y, Liu J, Wang Z, et al. Synthesis, properties, and optical applications of low-dimensional perovskites[J]. Chemical Communications, 2016, 52(94):13637.

[2]Bhuvaneshwari S, Gopalakrishnan N. Facile synthesis of low dimensional CuO nanostructures and their gas sensing applications[J]. Crystal Research \& Technology, 2016, 51(2):145-153.

[3]Bian Y, Gong Q. Optical performance of one-dimensional hybrid metal - insulator - metal structures at telecom wavelength[J]. Optics Communications, 2013, 308(11):30-35. 